
\title{
An XRD Study of Chemical Self-Discharge in Delithiated
Cobalt Oxide
}

\author{
Yasunori Ozawa, ${ }^{\mathrm{a}, \mathrm{b}, *}$ Rachid Yazami, ${ }^{\mathrm{a}, \mathrm{b}, * *, \mathrm{z}}$ and Brent Fultz ${ }^{\mathrm{a}, * *}$ \\ ${ }^{a}$ California Institute of Technology, Division of Engineering and Applied Science, Pasadena, \\ California 91125, USA \\ ${ }^{b}$ CNRS-UMR 5631, Grenoble, France
}

Changes in samples of $\mathrm{Li}_{1-x} \mathrm{CoO}_{2}$ were measured by X-ray diffractometry (XRD) after thermal aging treatments that cause capacity losses in electrochemical cells. Changes in lattice parameters were used to identify lithium re-intercalation into $\mathrm{Li}_{1-x} \mathrm{CoO}_{2}$ when it was aged in the presence of $\mathrm{LiClO}_{4}, \mathrm{LiPF}_{6}$, and $\mathrm{LiAsF}_{6}$ in propylene carbonate (PC). $\mathrm{Li}^{+}$re-intercalation could account for the reversible capacity loss. Thermal aging at $75^{\circ} \mathrm{C}$ in pure $\mathrm{PC}$ or pure argon gas resulted in other changes that are attributed to the formation of spinel phase. The rate of the lithium re-intercalation increases in the following sequence: $\mathrm{LiPF}_{6}<\mathrm{LiClO}_{4}<\mathrm{LiAsF}_{6}$.

(C) 2004 The Electrochemical Society. [DOI: 10.1149/1.1830395] All rights reserved.

Manuscript submitted December 26, 2003; revised manuscript received February 23, 2004. Available electronically November 29, 2004.

Lithium-ion batteries are now the dominant power source for high-technology portable electronic applications. They feature high energy density and excellent reliability. ${ }^{1}$ However, the aging mechanisms of these batteries are not well understood, including loss of capacity after extensive cycling, after exposure to high temperatures, or after over-charge and over-discharge. These important issues warrant a systematic study on the active components of the battery (e.g., the anode, cathode, and electrolyte), either singly or in combination. Useful results have already been obtained from such studies. For example, by knowing the activation energies of the processes that affect accelerated aging at elevated temperatures, the calendar life of batteries can be predicted at lower temperatures. ${ }^{2}$

Self-discharge is a natural phenomenon in primary and secondary batteries. ${ }^{3}$ It originates mainly from corrosion-type reactions that occur between the electrolytes and electrodes. Lithium-ion batteries in their charged state have a high open-circuit voltage close to $4.2 \mathrm{~V}$, which makes it even more likely for the lithiated graphite $\mathrm{Li}_{x} \mathrm{C}_{6}$ negative electrode (anode) and delithiated positive electrode (cathode) $\mathrm{Li}_{1-x} \mathrm{CoO}_{2}$ to be oxidized or reduced by the electrolyte, respectively. As a result, in half-cells with lithium counter electrodes, the open-circuit voltage $(\mathrm{OCV})$ will increase for $\mathrm{Li}_{x} \mathrm{C}_{6}$ and decrease for $\mathrm{Li}_{1-x} \mathrm{CoO}_{2}$ electrodes.

Recently we investigated the OCV and capacity loss of lithiated cobalt oxide using lithium half-cells of $\mathrm{LiCoO}_{2} \cdot{ }^{4,5}$ This study included the time dependence of reversible and irreversible capacity losses at elevated temperatures. We found that the thermal stability of delithiated (charged) cobalt oxide decreased with increased state of charge and increased temperature. We also showed the formation of the spinel phase as a result of aging for longer times and higher temperatures. We suggested that the hexagonal $\rightarrow$ cubic phase transition could account for the irreversible part of the capacity loss. For the reversible part of the capacity loss, we suggested that the lithium ions re-intercalate from the electrolyte in the delithiated cathode, with concomitant electrolyte oxidation. Although the $\mathrm{Li}^{+}$ion reintercalation is a more benign reversible reaction, the electrolyte oxidation will affect the cell performance because it may generate gases and may lead to increased internal impedance of the cell.

In the present study, we used X-ray diffractometry (XRD) to monitor the structural evolution of delithiated cobalt oxide during thermal aging at $75^{\circ} \mathrm{C}$ in different media: a neutral argon atmosphere, a pure liquid propylene carbonate (PC), and a $1 \mathrm{M}$ solution of $\mathrm{LiClO}_{4}, \mathrm{LiPF}_{6}$, and $\mathrm{LiAsF}_{6}$ in $\mathrm{PC}$. The purpose was to assess the role of each of the electrolyte components in the reversible and

\footnotetext{
* Electrochemical Society Student Member.

** Electrochemical Society Active Member.

z E-mail: yazami@caltech.edu
}

irreversible capacity losses. These are first steps, but important ones, for understanding and eventually controlling the self-discharge of $\mathrm{Li}$ ion batteries.

\section{Experimental}

$\mathrm{Li} / \mathrm{PC}-\mathrm{LiClO}_{4} / \mathrm{LiCoO}_{2}$ coin-type half-cells (CR2016) were used in this study. The cathode was provided by ENAX, Inc. (Tokyo, Japan). It consisted of a composite mixture of $91 \mathrm{wt} \% \mathrm{LiCoO}_{2}, 6$ wt $\%$ graphite, and $3 \mathrm{wt} \%$ binder, cast on a thin aluminum foil. The separator was made of glass fibers, provided by Crane Co. (New Hyde, NY, USA), Craneglas 230/6.1. The electrolyte was a molar solution of $\mathrm{LiClO}_{4}$ in propylene carbonate (PC) from Mitsubishi Chemicals Co. (Tsukuba, Japan). The cells were cycled at ambient temperatures at a C/5 rate between 2.9 and $4.2 \mathrm{~V}$ for several times. The final step was a charge to $4.2 \mathrm{~V}$. The cells were then disassembled in an argon-filled dry box. The positive electrode was removed, washed repeatedly with pure PC, dried, and then held in vacuum for several hours at ambient temperature. These cleaned electrodes were then aged at $75^{\circ} \mathrm{C}$ in different media: pure argon, $\mathrm{PC}$, and $1 \mathrm{M}$ solutions of PC-LiClO 4 , PC-LiPF 6 , and PC-LiAsF The aged electrodes were washed in $\mathrm{PC}$ and dried for several hours at ambient temperature. For XRD measurements with $\mathrm{CuK} \alpha$ radiation, the samples were protected from contact with air by sealing them in a hermetic cell. The (002) peak of graphite present in the electrode was used as internal reference to correct $2 \theta$ positions of the measured diffraction peaks.

\section{Results}

$\mathrm{LiCoO}_{2}$ has a layered rhombohedral structure $(R \overline{3} m$, space group 166). Its XRD pattern is characterized by a strong (003) peak. ${ }^{6}$ Depending on the state of charge " $x$ " in $\mathrm{Li}_{1-x} \mathrm{CoO}_{2}(0<x$ $<0.5)$, the crystal structure undergoes a series of phase transitions where the " $a$ " and " $c$ " crystal parameters of the hexagonal phase(s) vary with " $x$." For instance during charging, the (003) peak continuously shifts toward lower diffraction $2 \theta$ angles, corresponding to a nearly a linear increase of " $c$ " with " $x$ " from $14.08 \AA$ in $\mathrm{LiCoO}_{2}$ to $14.4 \AA$ in $\mathrm{Li}_{0.5} \mathrm{CoO}_{2}{ }^{6}$ This is illustrated in Fig. 1 where $\mathrm{LiCoO}_{2}$ was first charged to $4.2 \mathrm{~V}$ in a half-cell to form $\mathrm{Li}_{0.5} \mathrm{CoO}_{2}$. The cell was then discharged under a $\mathrm{C} / 5$ rate. XRD was performed ex situ after each hour of discharge. Figure 1 shows the progressive evolution of (003) and (006) peaks during re-lithiation. As expected, the two peaks shifted to higher angles. Moreover, the intensity of the (006) peak which could not be detected at the end of charge reappeared gradually at the end of discharge. XRD results can be used to determine the stoichiometry in $\mathrm{Li}_{1-x} \mathrm{CoO}_{2}$ by accurate measurement of the (003) peak position.

Figures $2 \mathrm{a}$ and $\mathrm{b}$ show, respectively, the evolution of the (003) and (006) peaks during thermal aging of $\mathrm{Li}_{0.5} \mathrm{CoO}_{2}$ in $\mathrm{PC}-\mathrm{LiClO}_{4}$. 


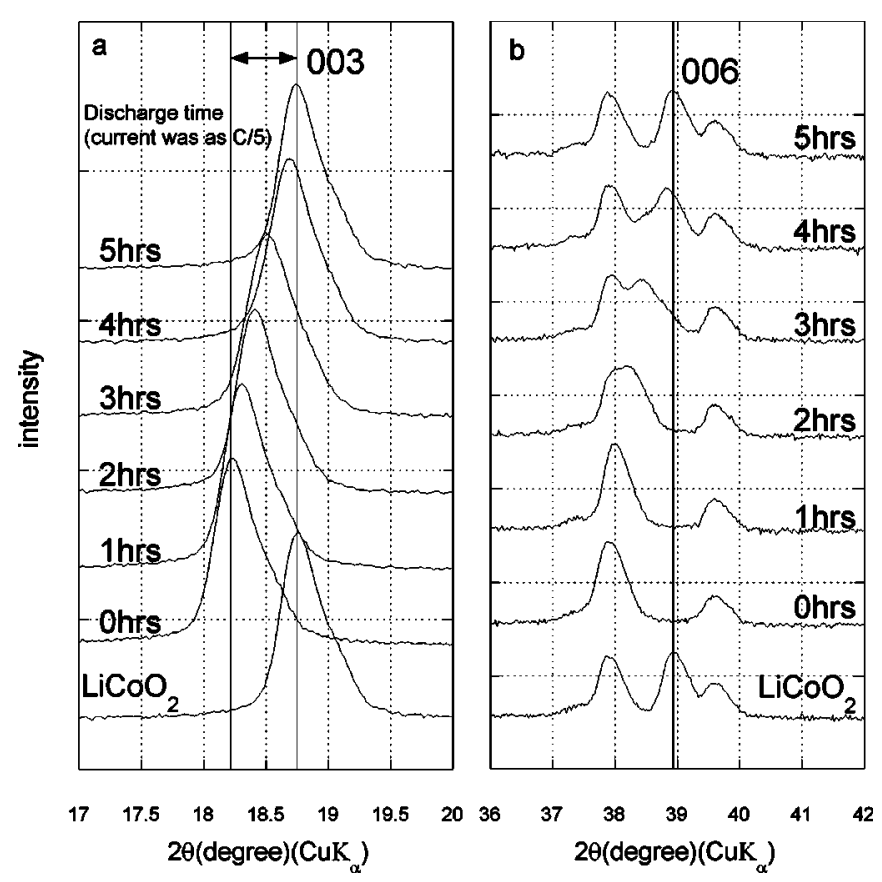

Figure 1. Time evolution of the (003) and (006) XRD peaks during electrochemical re-lithiation of $\mathrm{Li}_{0.5} \mathrm{CoO}_{2}(0 \mathrm{~h})$. At each hour, the Li composition is increased by 0.1 ( $\mathrm{C} / 5$ rate). The starting $\mathrm{LiCoO}_{2}$ material is shown for comparison.

The (003) peak shifted continuously to higher $2 \theta$ angles for the first 25 days or so. Then the shift rate slowed down for the next 25 days. The (006) peak re-appeared in its original position after 25 days, indicating that some of the original lithiated hexagonal structure may have been recovered.

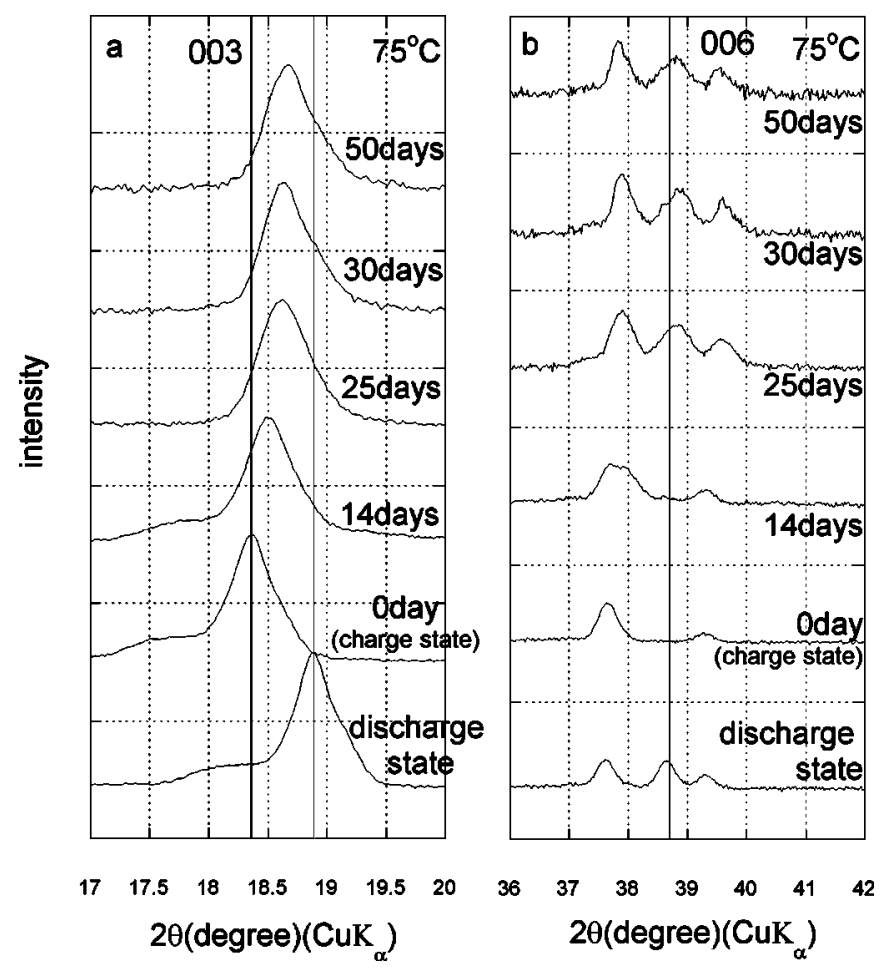

Figure 2. The evolution of the (003) and (006) XRD peaks $\left(\mathrm{CuK}_{\alpha}\right)$ during thermal aging of $\mathrm{Li}_{0.5} \mathrm{CoO}_{2}$ in $\mathrm{PC}-\mathrm{LiClO}_{4}$.

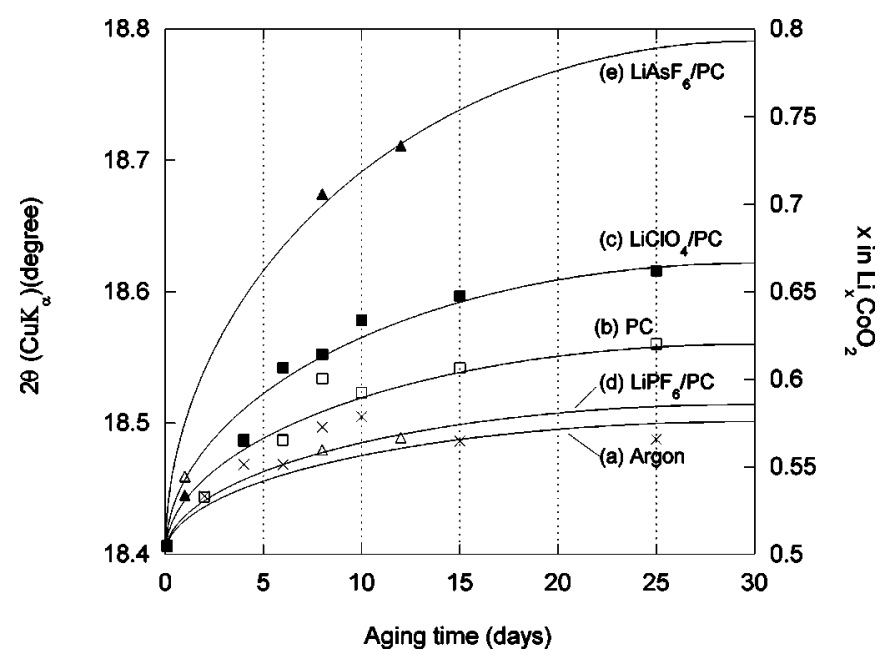

Figure 3. Comparison of the time dependence of the $2 \theta$ position and the $\mathrm{Li} / \mathrm{Co}$ ratio during aging of $\mathrm{Li}_{0.5} \mathrm{CoO}_{2}$ at $75^{\circ} \mathrm{C}$ in (a) argon, (b) $\mathrm{PC}$, (c) PC-LiClO 4 , (d) PC-LiPF 6 , and (e) PC-LiAsF 6 .

Figure 3 compiles results of aging kinetics of $\mathrm{Li}_{0.5} \mathrm{CoO}_{2}$ in argon (3a), PC (3b), PC-LiClO 4 (3c), PC-LiPF 6 (3d), and PC-LiAsF 6 (3e). The double $y$-axis presentation gives simultaneously the timedependence of the $2 \theta$ position of the (003) and of the electrode composition " $x$ " in $\mathrm{Li}_{x} \mathrm{CoO}_{2}$. The $2 \theta / x$ correspondence is obtained from the calibration curve in Fig. 1. It assumes the $2 \theta$ shift is caused by lithium re-intercalation only. This assumption does not apply for aging in argon and $\mathrm{PC}$ where no lithium is present. Aging in PC- $\mathrm{LiPF}_{6}$ and $\mathrm{PC}-\mathrm{LiAsF}_{6}$ was carried out for a shorter time (12 to 15 days) for comparison with $\mathrm{PC}-\mathrm{LiClO}_{4}$.

During aging in argon, the (003) peak remained unchanged in position for the first 12 days. It increased slightly during the following four weeks. The (006) peak did not return to its original position even after 39 days of aging. A similar trend was observed for the sample aged in pure PC. The (003) peak was unchanged in position for the first 10 days and then shifted only slightly upwards in $2 \theta$ angle. The shift in $2 \theta$ angle after 25 days in PC was larger than the one in argon after 32 days, indicating that PC itself may play a role in the aging process.

The kinetics of re-lithiation are quite different for aging in

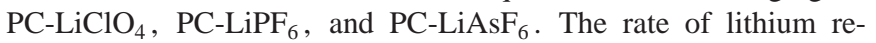
intercalation is found to increase in the following sequence: PC-LiPF 6 PC-LiClO 4 $<$ PC-LiAsF 6 . In PC-LiPF 6 , the change in the $\mathrm{Li} / \mathrm{Co}$ ratio was remarkably slow, almost the same as aging in $\mathrm{PC}$ only.

\section{Discussion}

Aging in argon, $\mathrm{PC}$, and $\mathrm{PC}-\mathrm{LiClO}_{4}$.- The changes in lattice parameters of lithium cobalt oxide during lithiation and delithiation are well documented in the literature. ${ }^{6,7}$ Starting with the discharged material of stoichiometry, $\mathrm{LiCoO}_{2}$, and delithiating to the fully charged material, $\mathrm{Li}_{0.5} \mathrm{CoO}_{2}$, the (003) peak shifts to lower diffraction angles. This indicates an increase in inter-layer spacing that accompanies $\mathrm{Li}^{+}$de-intercalation.

Regarding aging in PC and $\mathrm{PC}-\mathrm{LiClO}_{4}$, the largest effects were observed when the delithiated material was aged in $\mathrm{PC}-\mathrm{LiClO}_{4}$, where the (003) peak quickly shifted to higher diffraction angles. This shows that in $\mathrm{PC}-\mathrm{LiClO}_{4}, \mathrm{Li}^{+}$re-intercalates from the electrolyte. Electrons are needed to balance the charges for lithium intercalation. Electrons can be provided by $\mathrm{PC}$ oxidation or by the $\mathrm{ClO}_{4}^{-}$ transformation to a $\mathrm{ClO}_{4}^{\circ}$ radical. $^{8}$ The whole process of the lithium re-intercalation can be schematized as

$$
\mathrm{Li}_{0.5} \mathrm{CoO}_{2}+y \mathrm{Li}^{+}+y \mathrm{e}^{-} \rightarrow \mathrm{Li}_{0.5+y} \mathrm{CoO}_{2}
$$




$$
\left\{\begin{array}{cc}
y_{1} \mathrm{PC} \rightarrow y_{1} \mathrm{PC}^{+}+y_{1} \mathrm{e}^{-} & {[2]} \\
y_{2} \mathrm{ClO}_{4}^{-} \rightarrow y_{2} \mathrm{ClO}_{4}^{-}+y_{2} \mathrm{e}^{-} & {[3]}
\end{array} y=y_{1}+y_{2}\right.
$$

where $\mathrm{PC}^{+}$is a schematic representation of the oxidation products of PC as detailed in Ref. 9.

During aging in argon, the (003) peak remained unchanged in position for the first 2 weeks or so, and shifted a bit to higher angles after a longer storage time. This peak shift could be due to the formation of some spinel phase by thermal decomposition. Such a mechanism involves oxygen gas evolution, as reported by Graetz. ${ }^{10}$ A schematic reaction can be written as

$$
2 \mathrm{Li}_{0.5} \mathrm{CoO}_{2} \rightarrow \mathrm{LiCo}_{2} \mathrm{O}_{4(1-\varepsilon)}(\text { spinel })+2 \varepsilon \mathrm{O}_{2} \uparrow
$$

Our independent study by transmission electron microscopy showed that the cubic spinel co-exists with the H1-3 hexagonal phase characteristic of delithiated cobalt oxide. However, we did not find the $\mathrm{O} 3$ hexagonal phase of $\mathrm{LiCoO}_{2}$ predicted by Dahn et al. for the disproponation of $\mathrm{Li}_{0.5} \mathrm{CoO}_{2}$ to delithiated spinel and $\mathrm{LiCoO}_{2} .{ }^{11}$

In the case of aging in PC, Eq. 4 may still apply. We cannot rule out an additional process of oxidation of PC, as reported by Kanamura et al..$^{9}$ The PC oxidation may promote the hexagonal $\rightarrow$ spinel phase transformation on particle surfaces if the oxygen is supplied by the $\mathrm{LiCoO}_{2}$. Moreover, the $\mathrm{O}_{2}$ produced during thermal decomposition may react with $\mathrm{PC}$ to form $\mathrm{CO}_{2}$ and water. We observed a change in the color of the PC after aging, which we attribute to partial cobalt dissolution in PC. It is plausible, but not proved, that a dissolution/precipitation mechanism may also account for the surface chemistry in delithiated cobalt oxide during high temperature aging in PC.

In a recent transmission electron microscopy (TEM) study by our group, ${ }^{12}$ direct evidence of the cubic spinel phase formation on the surface of the hexagonal $\mathrm{Li}_{x} \mathrm{CoO}_{2}$ (H1-2 phase) was shown. As the spinel phase is known to have a lower electrochemical activity (i.e., lower discharge voltage and lower capacity) than the hexagonal phase ${ }^{13}$ this should account for the decrease in electrochemical performance of the $\mathrm{LiCoO}_{2}$ cathode upon thermal aging. Moreover, the hexagonal $\rightarrow$ spinel phase transformation is promoted by the delithiation (charged) state and by temperature, ${ }^{14}$ and is irreversible in nature.

It is known that electrochemical cells undergo capacity losses upon aging that are both reversible and irreversible. When the cell is operated normally after aging, the capacity of the cell is restored after recharging if the loss is reversible. Irreversible losses are also observed, where less lithium can be re-intercalated after aging. We note that $\mathrm{Li}^{+}$re-intercalation into the structure, as occurs in the presence of $\mathrm{LiClO}_{4}$, could be responsible for some of the reversible capacity loss. On the other hand, the formation of spinel phase is a major change in crystal structure, and is likely not reversible in the course of charge/discharge cycling. The formation of spinel phase could account for at least some of the irreversible capacity loss in $\mathrm{Li}_{1-x} \mathrm{CoO}_{2}$ cathodes.

Aging in PC-LiPF ${ }_{6}$ and PC-LiAs $F_{6}$ - - As shown in Fig. 3, the shift of the (003) peak in delithiated cobalt oxide during aging is larger in $\mathrm{PC}_{-} \mathrm{LiAsF}_{6}$ than in $\mathrm{PC}-\mathrm{LiClO}_{4}$ and is very low in $\mathrm{PC}-\mathrm{LiPF}_{6}$. These results show the important role played by the lithium salt anion in the chemical stability of the cathode material. Because the $\mathrm{P}$ and As belong to the same group in the periodic table, $\mathrm{LiPF}_{6}$ and $\mathrm{LiAsF}_{6}$ are expected to undergo similar dissociation, thermal decomposition, and redox reactions. For instance, upon dissociation in $\mathrm{PC}, \mathrm{LiAsF}_{6}$, and $\mathrm{LiPF}_{6}$ will thermally decompose, respectively, to $\mathrm{AsF}_{5}$ and $\mathrm{PF}_{5}$, which may then be followed by a series of acid-base and redox reactions eventually leading to the $\mathrm{Li}^{+}$reintercalation in $\mathrm{Li}_{0.5} \mathrm{CoO}_{2}$. These reactions may generate products or by-products with differences in solubility in PC, which will affect the chemistry taking place on the surface of delithiated cobalt oxide. In their work on the anodic oxidation on a nickel electrode of PC based electrolytes with different lithium salts, Kanamura et al. compared the amounts of decomposition products determined by Fourier transform infrared spectroscopy (FTIR) and the anodic current at 5 $\mathrm{V}$ determined by cyclic voltammetry (CV) according to the lithium salt. ${ }^{9}$ They found increasing anodic current in the following sequence: $\mathrm{LiBF}_{4}=\mathrm{LiPF}_{6}<\mathrm{LiAsF}_{6}<\mathrm{LiCF}_{3} \mathrm{SO}_{3}<\mathrm{LiClO}_{4}$. This sequence is different from ours as we found more lithium reintercalation in $\mathrm{LiAsF}_{6}$ than in $\mathrm{LiClO}_{4}$. The nature of the electrode may play a role in the rate of electrolyte oxidation. Aurbach et al. used XPS to investigate the chemical modifications on the surface of cycled $\mathrm{LiNiO}_{2}$ and $\mathrm{LiMn}_{2} \mathrm{O}_{4}$ electrodes in different electrolyte media including $\mathrm{LiPF}_{6}$ and $\mathrm{LiAsF}_{6} .{ }^{15}$ They reported important differences in the $\mathrm{C}_{1 \mathrm{~s}}$ and $\mathrm{F}_{1 \mathrm{~s}}$ spectra that suggest different surface chemistries with the two lithium salts. In an earlier work on the graphite/ electrolyte interface, we reported the enhanced stability achieved with $\mathrm{LiPF}_{6}$. We attributed this to the higher surface filming abilities of $\mathrm{PF}_{6}^{-}$compared to other anionic groups. ${ }^{16}$

In their study on the thermal stability of PC-based electrolytes with delithiated cobalt oxide, Yamaki et al. suggested the formation of a solid electrolyte inter-phase (SEI) on the surface of the metal oxide as a result of thermal decomposition of $\mathrm{LiPF}_{6} \cdot{ }^{17}$ Our results are in agreement with those of Yamaki et al. because a well passivating SEI would hinder the $\mathrm{Li}^{+}$re-intercalation. Moreover, Gee et al. showed that the thermal stability of $\mathrm{LiAsF}_{6}$-based electrolytes is reduced in an oxidative environment, ${ }^{18}$ which may explain the weaker protective role of the $\mathrm{LiAsF}_{6}$ based SEI and consequently the higher observed self-discharge rate.

Note that the kinetics curves obtained in $\mathrm{PC}-\mathrm{LiPF}_{6}$ and in argon almost coincide, as showen in Fig. $3 \mathrm{~d}$ and a, respectively. These curves fall below that in pure PC (Fig. 3b). This observation strongly supports the passivation model.

\section{Conclusion}

Using XRD, we studied changes in the crystal structures of samples of $\mathrm{Li}_{1-x} \mathrm{CoO}_{2}$ subjected to different aging treatments. Some of these aging treatments had been shown previously to be responsible for self-discharge and capacity loss of electrochemical cells. The changes in the (003) and (006) d-spacings were used to determine the lithium concentration of the material. The changes were found to vary strongly according to the aging media of $\mathrm{Li}_{1-x} \mathrm{CoO}_{2}$ at $75^{\circ} \mathrm{C}$. The largest structure change is attributed to lithium reintercalation that takes place when $\mathrm{Li}_{1-x} \mathrm{CoO}_{2}$ is aged in a PC based electrolyte solution. The rate of lithium re-intercalation increases as follows: $\mathrm{LiPF}_{6}<\mathrm{LiClO}_{4}<\mathrm{LiAsF}_{6}$.

Noticeable structure changes occurred even when no lithium salt was used in PC. These changes could be attributed to a hexagonal $\rightarrow$ spinel phase transition near the surface, perhaps promoted by the oxidation of PC. A smaller change took place as well during aging in pure argon gas. In this case the spinel phase transition is the more likely mechanism for the shift in the (003) peak position. Upon aging, electrode materials in lithium ion batteries are known to undergo reversible and irreversible capacity losses. We suggest that $\mathrm{Li}^{+}$re-intercalation into the $\mathrm{Li}_{1-x} \mathrm{CoO}_{2}$ structure accounts for at least some of the reversible capacity loss. In contrast, the spinel formation, which is irreversible in nature, should for the irreversible capacity loss.

It is interesting that the most popular salt for lithium ion batteries, $\mathrm{LiPF}_{6}$, provided the highest stability of delithiated cobalt oxide, as compared to $\mathrm{LiAsF}_{6}$ and $\mathrm{LiClO}_{4}$. The surface chemistry in relation with the thermal stability of these salts may be at the source of differences in the self-discharge kinetics. $\mathrm{LiPF}_{6}$ seems to yield a very stable protective film on the electrode surface.

\section{Acknowledgments}

The authors thank ENAX, Inc., Tokyo for providing the $\mathrm{LiCoO}_{2}$ electrode and for financial support and Mitsubishi Chemicals, Japan for providing the electrolyte. This work was supported by DOE through Basic Energy Sciences grant DE-FG03-00ER15035. 
The California Institute of Technology assisted in meeting the publication costs of this article.

\section{References}

1. Y. Nishi, in Lithium Ion Batteries, Fundamentals and Performance, M. Wakihara and O. Yamamoto, Editors, p. 181, Wiley-VCH, New York (1998).

2. M. Broussely, S. Heyrrere, P. Biensan, P. Katztejna, K. Nechev, and R. J. Staviewicz, J. Power Sources, 97-98, 13 (2001).

3. D. Linden, in Handbook of Batteries, 2nd ed., McGraw Hill, Inc., New York (1995).

4. Y. Ozawa, R. Yazami, and B. Fultz, J. Power Sources, 119-121, 918 (2003).

5. Y. Ozawa, R. Yazami, and B. Fultz, Abstract 116. The Electrochemical Society Meeting Abstracts, Vol. 2002-2, Salt Lake City, UT, Oct 20-24, 2002

6. T. Ozuku and A. Ueda, J. Electrochem. Soc., 141, 2972 (1994).

7. J. N. Reimers and J. R. Dahn, J. Electrochem. Soc., 139, 2091 (1992).

8. G. Cauquis and D. Serve, J. Electroanal. Chem., 24, 3 (1970).

9. K. Kanamura, S. Toriyama, S. Shiraishi, and Z. Takehara, J. Electrochem. Soc., 143, 2548 (1996)
10. J. Graetz, Ph.D. Thesis, California Institute of Technology, Pasadena, CA (2003).

11. J. R. Dahn, E. W. Fuller, M. Obrovac, and U. von Sacken, Solid State Ionics, 69 , 265 (1994)

12. H. Gabrisch, R. Yazami, and B. Fultz, Electrochem. Solid-State Lett., 5, A111 (2002).

13. R. J. Gummow, D. C. Liles, and M. M. Thackeray, Mater. Res. Bull., 28, 1177 (1993).

14. (a) A. Van der Ven, M. K. Aydinol, G. Ceder, G. Kresse, and J. Hafner, Phys. Rev. B, 58, 2975 (1998); (b) J. Reed, A. Van der Ven, and G. Ceder, Electrochem. Solid-State Lett., 4, A78 (2001).

15. D. Aurbach, K. Gamolsky, B. Markovsky, G. Salitra, Y. Goffer, U. Heider, R. Pesten, and M. Schmidt, J. Electrochem. Soc., 147, 1322 (2000).

16. R. Yazami, M. Deschamps, S. Genies, and J. C. Frison, J. Power Sources, 68, 110 (1997).

17. J.-I. Yamaki, Y. Baba, N. Katayama, H. Takatsuki, M. Egashira, and S. Okada, J. Power Soureces, 119-121, 789 (2003).

18. M. A. Gee and F. C. Laman, J. Electrochem. Soc., 140, L53 (1993). 